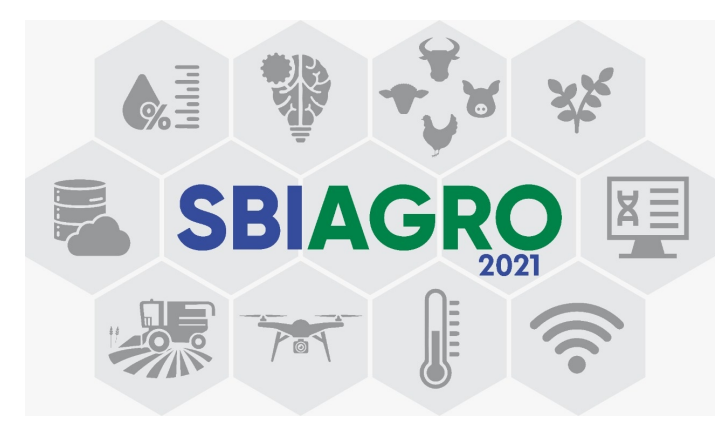

\title{
SQUADRO-i: Uma plataforma para o monitoramento da sustentabilidade de sistemas produtivos agropecuários
}

\author{
Kimberlly da Rosa Moreles ${ }^{1}$, Vinícius do Nascimento Lampert ${ }^{2}$, \\ Érico Marcelo Hoff do Amaral ${ }^{1}$ \\ ${ }^{1}$ Programa de Pós-graduação em Computação Aplicada \\ Universidade Federal do Pampa (UNIPAMPA) \\ Bagé - RS - Brasil \\ ${ }^{2}$ Embrapa Pecuária Sul (CPPSUL) \\ Bagé, RS - Brasil \\ kim.moreles@hotmail.com, vinicius.lampert@embrapa.br \\ erico.amaral@unipampa.edu.br
}

\begin{abstract}
The demand for sustainability assessments of agricultural systems has been growing in recent years. Based on a specific methodology for dairy production, a generic platform called SQUADRO-i has been developed, aimed at diagnosing the sustainability of different types of systems. This study presents the SQUADRO-i pilot project, a software that allows the construction of sustainability indicators and assessments. In the pilot project, it is already possible to build quantitative indicators with their respective parameters and calculate their sustainability index. The project is still in progress, but the possibility of the platform adapting to other assessment methodologies has already been raised.
\end{abstract}

Resumo. A demanda por avaliações de sustentabilidade de sistemas agropecuários tem sido crescente nos últimos anos. A partir de uma metodologia específica para a produção leiteira, têm-se desenvolvido uma plataforma genérica denominada SQUADRO-i, visando o diagnóstico de sustentabilidade de diferentes tipos de sistemas. Este estudo apresenta o projeto-piloto da SQUADRO-i, um software que permite a construção de indicadores e de avaliações de sustentabilidade. No projeto-piloto já é possível construir indicadores quantitativos com seus respectivos parâmetros e realizar o cálculo de índice de sustentabilidade dos mesmos. O projeto ainda se encontra em andamento, porém já levantou-se a possibilidade da plataforma se adaptar a outras metodologias de avaliação. 


\section{Introdução}

As preocupações com a sustentabilidade datam da década de 1970, porém foi o Relatório de Brundtland que trouxe a conceituação de desenvolvimento sustentável [Imperatives 1987]. Neste conceito, o desenvolvimento busca suprir as necessidades da geração atual sem prejudicar os recursos necessários às gerações futuras [Imperatives 1987, Bell e Morse 2012, das Nações Unidas ONU 2016], visando ir ao encontro das necessidades básicas humanas e possibilitando condições dignas de vida. Neste contexto, a agricultura possui um papel de destaque por demandar de grandes áreas para sua produção [Bell e Morse 2012] e por possuir um papel importante na economia brasileira, representando em torno de $20 \%$ do PIB nacional [de Oliveira e Carraro 2019].

Nos últimos anos, a demanda por avaliações de sustentabilidade de sistemas agropecuários tem sido crescente e para esta tarefa popularizou-se o uso de indicadores e índices de sustentabilidade. Os indicadores de desenvolvimento sustentável são instrumentos que medem as modificações de um sistema [Deponti et al. 2002] e precisam ser construídos de forma a contribuir para uma sustentabilidade autorregulada [Sitarz 1993]. A partir deles é possível determinar a necessidade de mudanças na condução de uma determinada estratégia ou até mesmo comparar o desempenho deste indicador em diferentes localidades e contextos. Para esta comparação, empregam-se índices que normalizam esses indicadores a uma mesma unidade de medida e que quando construídos com dados relevantes são capazes de inclusive servir de referência para o direcionamento de investimentos [Negra et al. 2020]. As avaliações construídas precisam levar em consideração não somente as características inerentes do sistema produtivo, como também os objetivos dos avaliadores. Neste sentido, apesar de existirem amplas possibilidades de metodologias de avaliação, ainda há uma escassez de ferramentas que se adaptem para o diagnóstico do desenvolvimento sustentável de sistemas produtivos e que gerem informações relevantes de forma eficaz e não onerosa.

O objetivo desta pesquisa é propor uma plataforma genérica que por meio de uma ferramenta computacional auxilie na sistematização de indicadores de sustentabilidade, para que desta forma os diagnósticos dos diferentes sistemas possam ser feitos conforme suas características e necessidades. A plataforma em construção, nomeada SQUADRO-i, se propõe a ser um ambiente onde os usuários poderão construir avaliações de sustentabilidade personalizadas, onde também será possível gerar um formulário de coleta de dados a campo que estará disponível para acesso em smartphones. A partir desta coleta, a SQUADRO-i disponibilizará o resultado não somente do índice geral da avaliação, como também os índices dos indicadores e dimensões avaliadas, podendo estas informações serem disponibilizadas a demais interessados autorizados pelo gerenciador da avaliação.

Em uma perspectiva técnica este trabalho visa contribuir para que o ambiente avaliativo seja adaptável, sendo possível delimitar as variáveis que melhor definirem o sistema estudado, sem ser necessário construir uma nova ferramenta específica para cada diagnóstico, e também, proporcionar um ambiente de coleta de dados dinâmico via aplicação móvel. Já em um panorama científico, este estudo visa criar algoritmos específicos para o cálculo de índices de indicadores e também para o cálculo de demais índices necessários à avaliação.

Neste artigo são dispostos os resultados obtidos até o momento com a plataforma SQUADRO-i, e as próximas etapas a serem concluídas e perspectivas futuras de estudo 
com a mesma. Para tal, na seção 2 é descrita a metodologia deste estudo, na seção 3 é apresentado o referencial teórico, na seção 4 é expressa a proposta da plataforma e os resultados já alcançados, e por fim, na seção 5 são elencadas as conclusões obtidas até o momento.

\section{Metodologia}

Quanto a sua natureza a pesquisa é classificada como pesquisa aplicada, pois pretende resolver um problema específico do desenvolvimento sustentável quanto a utilização de um sistema computacional para a sua avaliação. Empregou-se o método dedutivo, pois é pretendido avaliar a capacidade da ferramenta construída gerenciar o processo de avaliação de indicadores de forma genérica. Quanto aos seus objetivos a pesquisa é classificada como descritiva por pretender analisar o comportamento da ferramenta diante indicadores que avaliam diferentes sistemas produtivos agropecuários. Do ponto de vista da forma de abordagem do problema ela é classificada como quantitativa devido à plataforma manipular valores numéricos e fórmulas. Quanto aos procedimentos técnicos a pesquisa é classificada como bibliográfica, documental e experimental.

As etapas empregadas na condução da pesquisa até o momento e que levam à construção do projeto-piloto seguem a linha do tempo apresentada na Figura 1. Determinou-se que inicialmente, seguindo a engenharia de requisitos, seriam realizadas entrevistas junto a um profissional da Embrapa para a delimitação do problema de pesquisa e para elencar os requisitos necessários à solução. Durante estas, ficou convencionado o uso da metodologia mencionada no estudo de [Brum et al. 2017] como base para a implementação da solução, a mesma teve como objetivo o diagnóstico do desenvolvimento sustentável de propriedades familiares com atividade leiteira. Esta possui uma planilha Excel que foi utilizada como auxílio não só para o desenvolvimento, como também para a aplicação de testes na SQUADRO-i. O profissional entrevistado foi o responsável pelo desenvolvimento da metodologia modelada na planilha eletrônica. A fórmula de interpolação linear utilizada nesta planilha para o cálculo do índice dos indicadores também serviu como base para o algoritmo de mesma função na plataforma SQUADRO-i.

Após a etapa das entrevistas, determinou-se que iriam ser realizadas pesquisas abrangendo tanto a temática de indicadores quanto a de diagnósticos de sustentabilidade. Para a etapa seguinte ficou especificada a escrita de um documento de requisitos, contendo a modelagem do sistema, sua arquitetura e seus requisitos, e a partir deste documento, a construção de protótipos de tela necessários à validação dos requisitos. Por fim, levantou-se a necessidade de implementação de um projeto-piloto da plataforma para que se pudesse verificar a possibilidade de implementação das funcionalidades iniciais e cálculos essenciais em tempo hábil. Foi dado seguimento à implementação da aplicação e nela codificado o algoritmo de cálculo de índice dos indicadores, este calculando o índice com base nos parâmetros definidos para o dito indicador, sendo que qualquer valor intermediário aos parâmetros especificados é calculado para um valor aproximado, ou seja, uma interpolação linear. Para que as funcionalidades do projeto-piloto da plataforma fossem validadas, os resultados do mesmo foram comparados com os obtidos na ferramenta Excel. Para tal, procedeu-se a construção da cópia de um indicador, constante na ferramenta Excel, na plataforma SQUADRO-i, contendo a mesma estrutura e parâmetros de cálculo de índice, e posteriormente injetado no banco de dados o valor que este indicador 


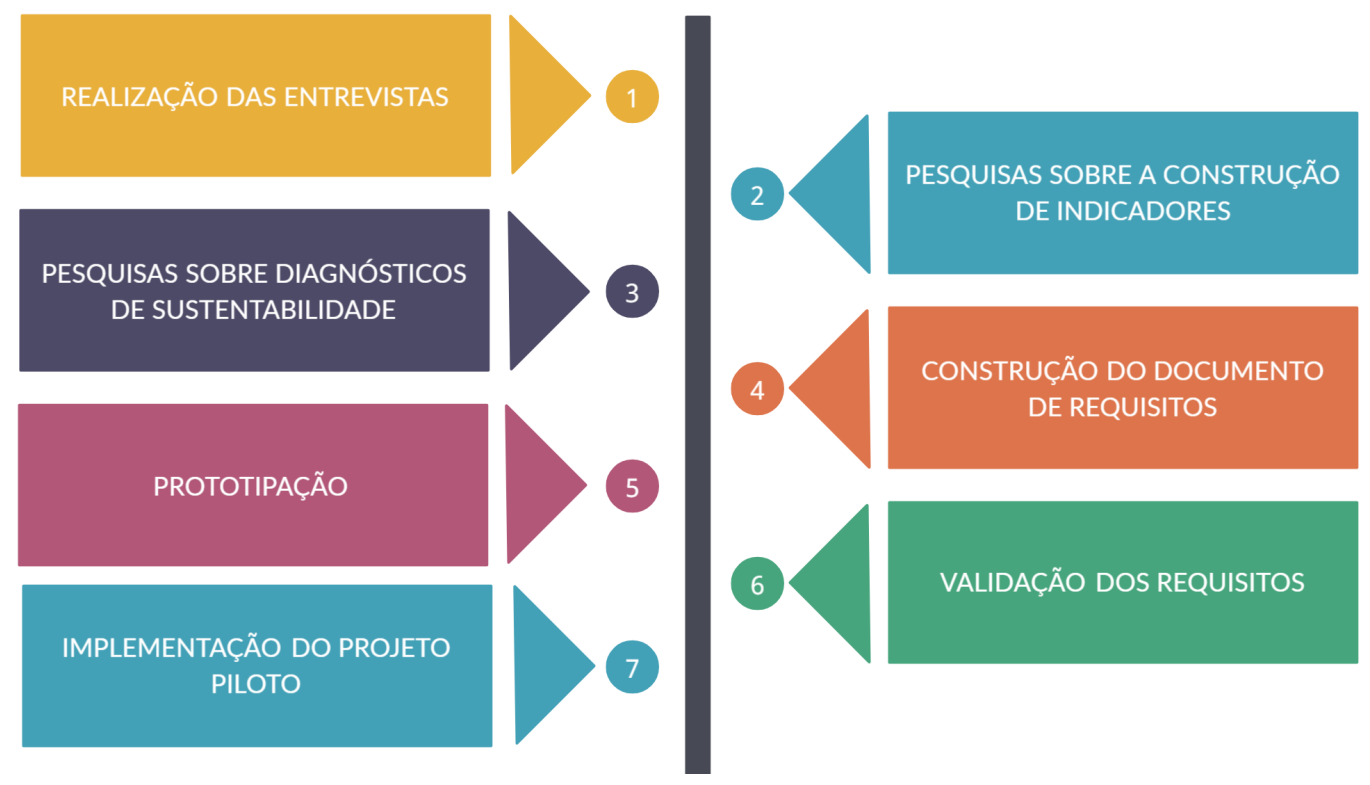

Figura 1. Etapas da pesquisa

apresentou na coleta de dados a campo para uma unidade avaliativa aleatória.

\section{Referencial Teórico}

A sustentabilidade engloba a capacidade de um sistema utilizar recursos de forma que este não seja perdido ou venha a escassear. Já o desenvolvimento sustentável faz menção ao uso de recursos no presente de forma que este uso não afete as necessidades das gerações futuras [Imperatives 1987]. Quando se fala neste último é necessário levar em consideração as dimensões ambiental, econômica e social [Bell e Morse 2012], logo é imprescindível que estes aspectos estejam presentes na construção de uma avaliação de sustentabilidade.

Ao se estudar um determinado sistema agrícola ou outro sistema qualquer quanto a sua sustentabilidade é necessário levar em consideração não somente estes aspectos, como também as características inerentes deste sistema. Estes aspectos, ou dimensões, por vezes são complementados por outras como, por exemplo, a dimensão produtiva e a dimensão institucional, a depender dos objetivos da avaliação em seguimento.

Outra característica denotada como importante para a construção de uma avaliação de sustentabilidade é a temporalidade. Segundo [López-Ridaura et al. 2002], este tipo de avaliação só pode se dar de forma comparativa e a comparação temporal é uma das alternativas. Para [Zhen e Routray 2003] é importante avaliar não somente práticas específicas empregadas no contexto da fazenda com o uso de indicadores adequados, como também a janela de tempo em que a avaliação irá ocorrer. Assim, este tipo de avaliação necessita não somente de um objetivo claro de avaliação e de quais informações são necessárias, como também a especificação do período de tempo em que a mesma irá ocorrer.

Em relação aos indicadores envolvidos na avaliação vale ressaltar que existe a demanda de que estes reflitam o grau de sustentabilidade dos sistemas produtivos [López-Ridaura et al. 2002], porém grandes quantidades dos mesmos não denotam qualidade na avaliação. A seleção de indicadores está diretamente relacionada ao sistema a 
ser avaliado e a região que a avaliação irá abranger tornando cada avaliação única, com poucas possibilidades de ser reaproveitada sem adaptações.

Há amplas possibilidades de metodologias de diagnóstico de sustentabilidade que são aplicáveis a sistemas produtivos agropecuários [Bohanec e Rajkovic 1990, Astier et al. 1999]. Destas metodologias, algumas contam com ferramentas computacionais específicas para uso, já outras necessitam da implementação de um sistema ou elaboração de uma planilha eletrônica.

Observa-se na literatura [Rodrigues et al. 2016, Lampert et al. 2018] que planilhas eletrônicas são utilizadas com certa frequência para diagnósticos de sustentabilidade, isto devido à flexibilidade proporcionada pelas mesmas em relação à adaptabilidade. Porém, também é observado que para este tipo de ferramenta há a necessidade de realização das coletas em formulários, e em uma etapa posterior, manualmente realizar a inserção dos dados. Também foram encontrados trabalhos que empregam sistemas computacionais [Chen et al. 2014, Lampert et al. 2015, Rezaei et al. 2018, Whitehead et al. 2020], alguns destes com soluções específicas e outros com a proposta de flexibilizar o processo avaliativo. Nestes últimos as principais limitações encontradas foram: uma seleção fechada de opções de personalização da avaliação e possibilidade de tratar apenas características qualitativas.

\section{Proposta e Resultados}

Na maioria das vezes, a construção de uma ferramenta de diagnóstico de sustentabilidade na agricultura envolve diversos interessados, gerando uma certa complexidade no processo de criação da mesma [Whitehead et al. 2020]. Envolver o usuário na construção, torna o sistema mais compreensível, adaptável e com mais chances de ser melhorado, sendo necessário que a usabilidade do mesmo seja garantida [Goumopoulos e Mavrommati 2020]. Porém, a complexidade de utilização é um fatorchave quando se fala na adesão desta ferramenta [Goumopoulos e Mavrommati 2020]. Ferramentas de avaliação podem abranger propósitos diversos e possuírem abordagens distintas [Ness et al. 2007], mas são as pessoas envolvidas na construção da ferramenta de sustentabilidade e as funcionalidades desenvolvidas para a mesma que irão definir o seu nível de sucesso [Goumopoulos e Mavrommati 2020].

Visando suprir a necessidade de construção de uma ferramenta específica para cada diagnóstico a ser realizado, neste estudo é apresentada a solução denominada SQUADRO-i. Esta irá possuir dois módulos: um módulo web contendo a parte gerencial de construção de uma avaliação de sustentabilidade e a possibilidade de visualização de resultados da avaliação em relatórios e gráficos, e um módulo móvel dedicado à coleta de dados a campo, substituindo assim a necessidade de formulários de papel.

A plataforma SQUADRO-i tem por base a metodologia mencionada por [Brum et al. 2017], onde uma avaliação é composta por dimensões e esta última por indicadores. Tanto estes, quanto as dimensões são ponderados por pesos, para que desta forma seja possível determinar indicadores e dimensões mais ou menos expressivos que os demais. Cada indicador também irá estar atrelado a um conjunto de parâmetros, estes serão o conjunto de regras que serão aplicadas para o cálculo de interpolação linear de seu índice. A estrutura completa de uma avaliação pode ser visualizada na Figura 2.

O presente trabalho teve início com a realização de entrevistas junto a um pro- 


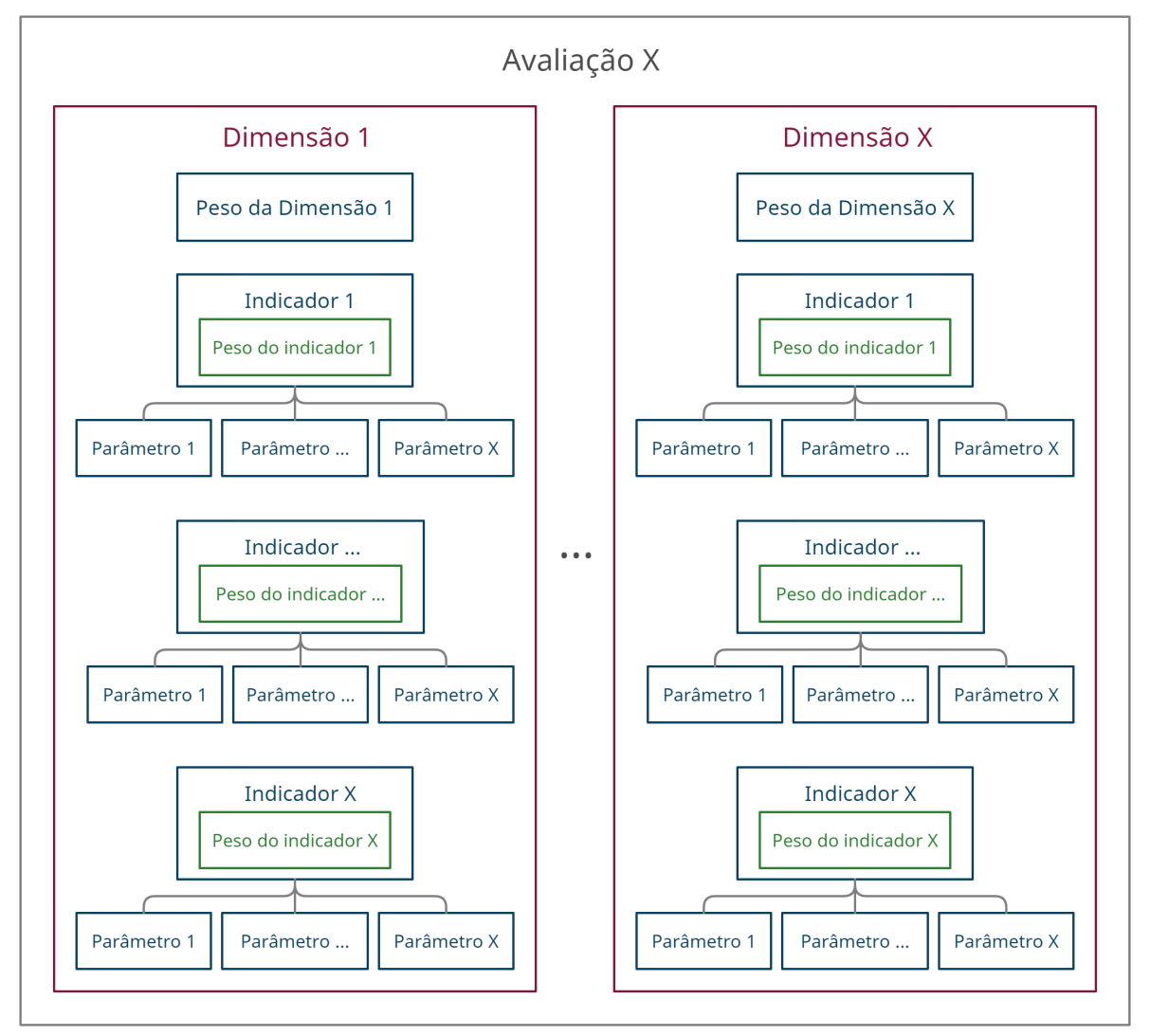

Figura 2. Estrutura da avaliação

fissional da Embrapa para que os requisitos necessários à plataforma fossem elencados. Logo após, para que a SQUADRO-i atenda adequadamente às necessidades levantadas, foram realizadas pesquisas bibliográficas e documentais sobre a temática de indicadores e de diagnóstico de sustentabilidade. Com estas informações, foi possível a construção de um documento de requisitos contendo a modelagem e arquitetura do sistema.

Em seguida, foi construído um protótipo da aplicação web para que os requisitos pudessem ser validados e a partir deste, iniciada a implementação do projeto-piloto que abrange as funcionalidades de inserção de indicadores quantitativos simples, componentes e parâmetros na SQUADRO-i, e também o algoritmo de cálculo do índice dos indicadores quantitativos simples. A partir do estudo e análise das tecnologias disponíveis no mercado, definiu-se como plataforma para a construção da solução web a linguagem PHP aliada às funcionalidades do JavaScript e o PostgreSQL para o gerenciamento do banco de dados.

Nesta etapa da pesquisa, a fim de validar o projeto-piloto, foram utilizados os dados e resultados da planilha Excel da metodologia mencionada por [Brum et al. 2017]. Para tal, comparou-se o resultado do cálculo do índice do indicador na SQUADRO-i com o resultado para o mesmo cálculo na planilha original do Excel.

Para apresentação desta fase da ferramenta elencou-se o indicador "POTENCIAL PRODUTIVO" e a unidade avaliativa "Nome 57". Na Figura 3 é apresentado este indicador na planilha Excel. Como podemos ver, ele é avaliado por uma única variável, esta 
chamada "Área Total”, que por sua vez possui valor 10 para a unidade avaliativa "Nome 57". Também é possível observar na Figura 3 quais os parâmetros de notas utilizados para o cálculo do índice deste indicador, que para o valor 10 recebeu índice 0,30 .

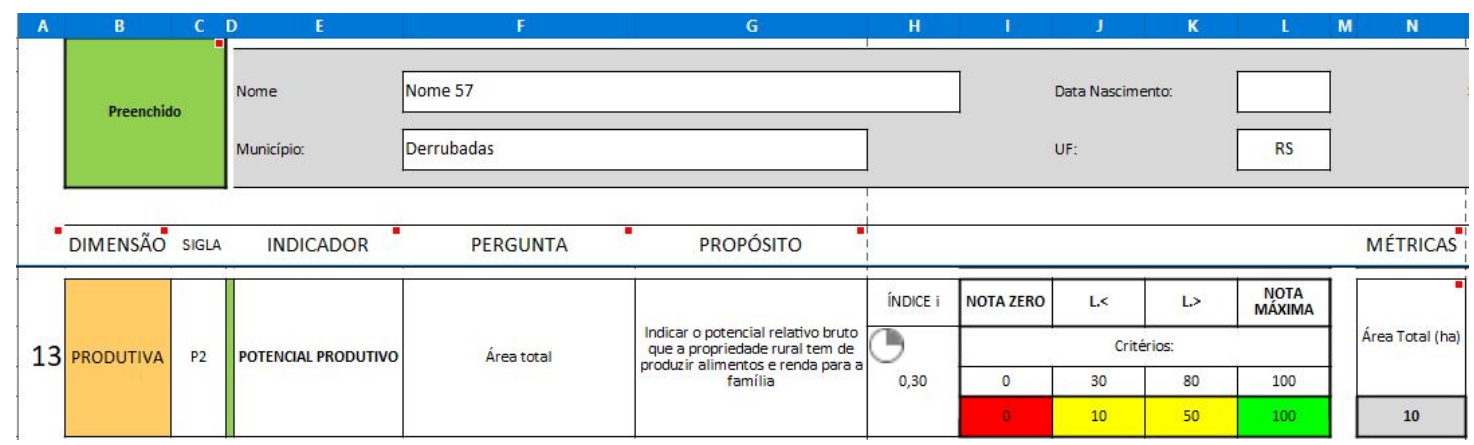

Figura 3. $O$ indicador "POTENCIAL PRODUTIVO" para a unidade avaliativa "Nome 57" na ferramenta Excel da metodologia SQUADRO

Na plataforma SQUADRO-i procedeu-se à inserção do indicador "POTENCIAL PRODUTIVO", a atribuição dos mesmos parâmetros de cálculo de índice, presentes na planilha Excel para este, e a injeção dos mesmos valores recebidos pela unidade avaliativa "Nome 57" na coleta de dados a campo. Na Figura 4 é apresentado o indicador "POTENCIAL PRODUTIVO" com valor 10 e seu índice já calculado pelo clique no botão "Calcular índice". Nesta etapa o indicador obteve índice 0,3, o mesmo resultado obtido na planilha Excel.
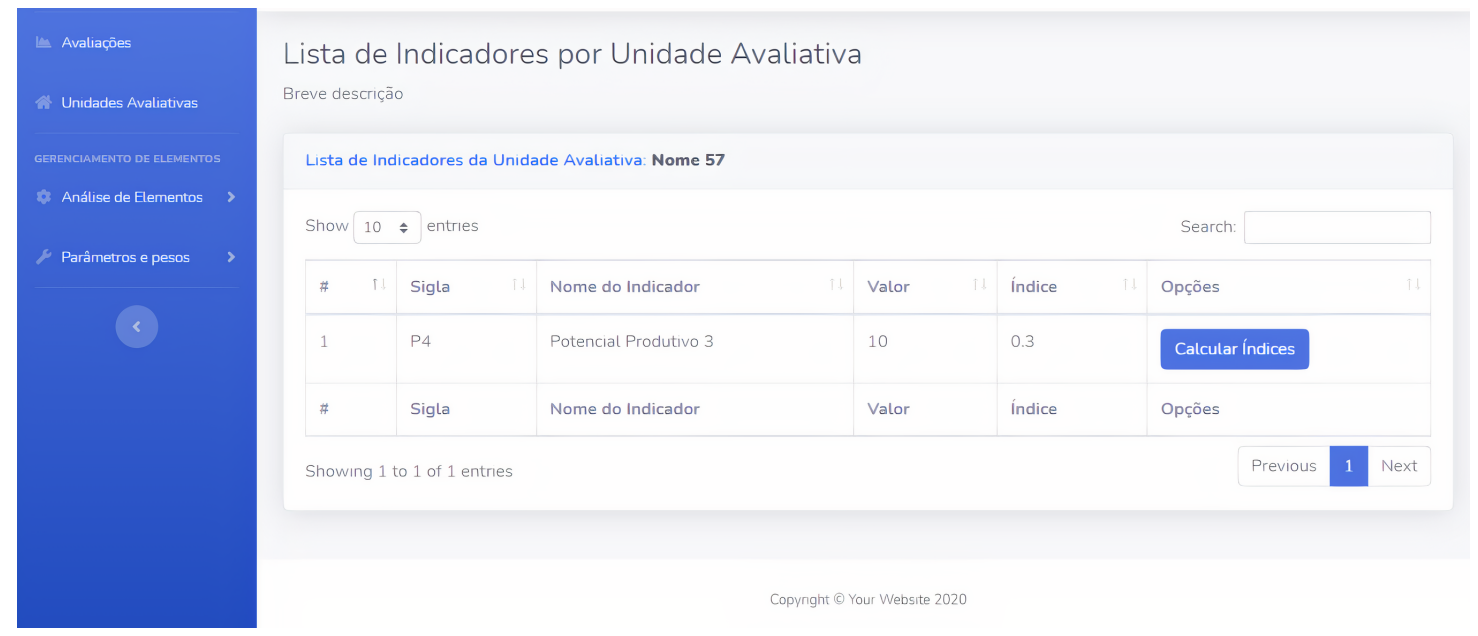

Figura 4. $O$ indicador "POTENCIAL PRODUTIVO" para a unidade avaliativa "Nome 57" na plataforma SQUADRO-i

Visto que o trabalho ainda se encontra em andamento e que o tipo de indicador testado não engloba todas as possibilidades necessárias à construção de uma avaliação de sustentabilidade, nas próximas etapas serão construídos e testados indicadores qualitativos e indicadores resultantes de fórmulas.

\section{Conclusões}

A construção de um sistema de avaliação de sustentabilidade é uma tarefa complexa que precisa envolver diferentes interesses e no contexto agropecuário envolver também as 
necessidades do produtor rural. As ferramentas disponíveis, em geral, são construídas especificamente para o diagnóstico a ser realizado, demandando tempo também para a implementação da mesma. A SQUADRO-i é uma ferramenta que tem a proposta ser customizável para diferentes tipos de avaliação. Para tal, ela irá contar com um módulo web onde será possível construir e consultar resultados de uma avaliação de sustentabilidade e um módulo móvel que possibilitará a coleta de dados a campo sem a necessidade de formulários de papel.

Apesar da complexidade envolvida no processo de diagnóstico de sustentabilidade e de ser um projeto ainda em andamento, a plataforma já foi capaz de realizar o cálculo do índice dos indicadores, por meio de uma interpolação linear, para indicadores do tipo quantitativo simples. Os passos futuros envolvem a implementação das demais funcionalidades da ferramenta e a adequação da mesma para suportar outros tipos de indicadores.

A plataforma SQUADRO-i é adaptável a diversos tipos de avaliação, pois a mesma foi criada na intenção de ser genérica e customizável para diferentes cenários avaliativos. Inicialmente ela está sendo testada no monitoramento da sustentabilidade de sistemas produtivos agropecuários, porém nada impede que a mesma seja aplicada em outras áreas. É pretendido verificar também a possibilidade de esta plataforma ser capaz de se adequar a outras metodologias de avaliação como, por exemplo, a metodologia MESMIS [Astier et al. 1999, López-Ridaura et al. 2002].

Por fim, os autores agradecem o suporte financeiro fornecido pela Coordenação de Aperfeiçoamento de Pessoal de Nível Superior (CAPES) e pelo programa de auxílio da pós-graduação (PAPG) da Universidade Federal do Pampa (UNIPAMPA).

\section{Referências}

Astier, M., Masera, O., e Lopez-Ridaura, S. (1999). Sustentabilidad y manejo de recursos naturales: El Marco de evaluación MESMIS.

Bell, S. e Morse, S. (2012). Sustainability indicators: measuring the immeasurable? Routledge.

Bohanec, M. e Rajkovic, V. (1990). DEX: An expert system shell for decision support. Sistemica, 1:145-157.

Brum, L. d. L., Lampert, V. d. N., Camargo, S. d. S., e Eickhoff, F. (2017). Aplicação de técnicas de mineração em dados de propriedades leiteiras do município de DerrubadasRS. In Embrapa Pecuária Sul-Artigo em anais de congresso (ALICE). In: SIMPÓSIO DA CIÊNCIA DO AGRONEGÓCIO, 5., 2017, Porto Alegre.

Chen, D., Thiede, S., Schudeleit, T., e Herrmann, C. (2014). A holistic and rapid sustainability assessment tool for manufacturing SMEs. CIRP Annals, 63(1):437 - 440.

das Nações Unidas ONU, O. (2016). A agenda 2030 para o desenvolvimento sustentável.

de Oliveira, E. C. e Carraro, N. C. (2019). Análise do comportamento e participação do agronegócio na composição do produto interno bruto (PIB) brasileiro: Um estudo da série temporal de 1996 a 2017. Brazilian Journal of Development, 5(11):24042-24064.

Deponti, C. M., Eckert, C., e Azambuja, J. d. (2002). Estratégia para construção de indicadores para avaliação da sustentabilidade e monitoramento de sistemas. Agroecologia e desenvolvimento rural sustentável, 3(4):44-52. 
Goumopoulos, C. e Mavrommati, I. (2020). A framework for pervasive computing applications based on smart objects and end user development. Journal of Systems and Software, 162:110496.

Imperatives, S. (1987). Report of the world commission on environment and development: Our common future. 10.

Lampert, V. d. N., da Silva, G., e Weiller, O. (2018). Nova planilha de sistematização da produção (Nova PSP): ferramenta de apoio no diagnóstico e intervenção em unidades de produção familiar com atividade leiteira. Embrapa Pecuária Sul-Documentos (INFOTECA-E).

Lampert, V. d. N., do Amaral, E., Lopes, P. P., Fracari, J. R., Dantas, T. F., e Oliveira, M. (2015). Uma ferramenta para gestão de indicadores na produção de bovinos de corte: simplificando a organização de processos. In Embrapa Pecuária Sul-Artigo em anais de congresso (ALICE). In: CONGRESSO BRASILEIRO DE AGROINFORMÁTICA, 10., 2015, Ponta Grossa.

López-Ridaura, S., Masera, O., e Astier, M. (2002). Evaluating the sustainability of complex socio-environmental systems: the MESMIS framework. Ecological Indicators, 2(1):135 - 148. Hyatt S.I.

Negra, C., Remans, R., Attwood, S., Jones, S., Werneck, F., e Smith, A. (2020). Sustainable agri-food investments require multi-sector co-development of decision tools. Ecological Indicators, 110:105851.

Ness, B., Urbel-Piirsalu, E., Anderberg, S., e Olsson, L. (2007). Categorising tools for sustainability assessment. Ecological Economics, 60(3):498-508.

Rezaei, M. E., Barmaki, M., e Veisi, H. (2018). Sustainability assessment of potato fields using the DEXi decision support system in Hamadan Province, Iran. Journal of Integrative Agriculture, 17(11):2583 - 2595.

Rodrigues, G. S., Barros, I. d., e Martins, C. (2016). Análise de sustentabilidade em diferentes sistemas de produção de coco no norte e nordeste. In Embrapa Tabuleiros Costeiros-Artigo em anais de congresso (ALICE). In: SEMINÁRIO DE INTENSIFICAÇÃO ECOLÓGICA DA FRUTICULTURA TROPICAL, 4.

Sitarz, D. (1993). Agenda 21: The earth summit strategy to save our planet.

Whitehead, J., MacLeod, C. J., e Campbell, H. (2020). Improving the adoption of agricultural sustainability tools: A comparative analysis. Ecological Indicators, 111:106034.

Zhen, L. e Routray, J. K. (2003). Operational indicators for measuring agricultural sustainability in developing countries. Environmental Management, 32(1):34-46. 